Ann. Zootech., I964, 13 (4), 355-366.

\title{
ESSAI D'ESTIMATION DIRECTE DES DIFFÉRENTS ÉLÉMENTS DE LA CARCASSE DU POULET EN VUE D'APPRÉCIER SON RENDEMENT EN VIANDE
}

\author{
F. II. RICARD \\ Station expérimentale d'Aviculture du Magneraud, \\ Saint-Pierre-d'Amilly (Charente Maritime)
}

SOMMAIRE

Trois coquelets et 3 poulettes de 2 souches différentes (dont une de type Cornish) ont été abattus à I I semaines et disséqués entièrement. En moyenne, les cuisses et pilons représentent 24 p. Ioo du poids vif, les ailes $8 \mathrm{p}$. 100, les blancs I4 p. 100, le reste de carcasse I9 p. I00, les abats consommables $4 \mathrm{p}$. I00, la graisse abdominale I p. Ioo, les pertes à l'abattage et à l'éviscération $30 \mathrm{p}$. 100. La carcasse éviscérée contient i 7 p. Ioo d'os, 69 p. Ioo de viande et ro p. Ioo de peau. Le total des éléments consommables représente environ les $2 / 3$ de la carcasse effilée.

On observe un meilleur rendement a l'abattage pour la souche Cornish et pour les coquelets. Par rapport au poids éviscéré, les éléments de la carcasse présentent une variabilité relativement faible entre souches, un peu plus nette entre sexes. Quand on compare les souches, la répartition de la viande sur la carcasse est plus variable que le pourcentage total de cette viande, le type Cornish ayant la meilleure répartition (blancs plus développés). Les poulettes avec moins d'os et plus de blancs offrent au consommateur une carcasse plus intéressante que celle des coquelets.

\section{INTRODUCTION}

L'étude de la composition anatomique d'un animal de boucherie et de ses variations permet de préciser l'importance relative des éléments consommables et non consommables et peut orienter la sélection de caractères tels que le rendement en viande. De nombreux travaux ont été réalisés sur les mammifères, en particulier par l'équipe de Hammond (voir Paisson, I955). Boccard et Dumont (I960) et Dumont et al. (I96I) ont entrepris l'étude de la production de viande chez les ovins et les bovins.

Chez les volailles, la production de viande est assurée en grande partie par des 
souches spécialisées, les souches " chair ", pour lesquelles un bon rendement en viande constitue une qualité recherchée par le consommateur. C'est pourquoi plusieurs auteurs ont cherché à préciser l'importance et les variations des éléments consommables présents sur une carcasse. Lres méthodes utilisées peuvent se diviser en 3 groupes :

Io La méthode la plus simple consiste à n'étudier qu'un nombre réduit d'os, de muscles ou d'organes. Ainsi DELPECH et GoUTERON (I962) travaillent sur 2 muscles (Pectoralis profondus et Gastrocnemius), un os (Tibiotarsus) et 2 organes (foie et testicule). D'autres auteurs apprécient le rendement en viande d'après l'ensemble blanes + viande des cuisses et pilons (OKERMAN, I960) ou les blanes seuls (FRISCHKNECHT et JULI, I946).

$2^{0}$ Une technique relativement rapide consiste à séparer les tissus après cuisson de la carcasse. C'est le cas des travaux de HeNDERSon (I946), STotTs et Darrow (I953), Morrison et al. (I954), ORR (I955), Haverman et al. (I957), Péters (I958), Dawson et al. (I958), Gilpin et al. (I960), Kondra et al. (I962). Nous-mêmes avons étudié de cette façon l'importance du squelette (RICARD, Ig6r). Mais les résultats obtenus intègrent la variabilité due au mode de cuisson, aux pertes de substance et aux modifications qui en résultent.

$3^{\circ}$ La meilleure méthode, mais aussi la plus longue, consiste à disséquer directement et entièrement la carcasse crue. C'est ce qu'ont fait MrTCHEL, et al. (Ig26 et I93I), Harshaw (I938), Maw et Maw (I939), Poley et al. (I940), Brown et Bean (I952), Hahaway et al. (I953). Wilson (I954) combine la dissection directe et des traitements physico-chimiques pour obtenir le poids frais et la matière sèche des éléments de la carcasse.

Nous avons essayé la méthode des dissections complètes sur I2 poulets âgés de I s semaines. Nous voulions apprécier les avantages et les inconvénients de la méthode et avoir une idée personnelle et précise de la composition anatomique d'un poulet.

\section{MATÉRIEL ET MÉTHODES}

Les I 2 poulets se répartissaient en 3 coquelets et 3 poulettes d'une souche $A$, de type Cornish, et d'une souche B, obtenue à partir des races Bresse blanche et Now-Hamsphire. Ia souche B se caractérise par une vitesse de croissance nettement inférieure, un angle de poitrine plus faible et un corps plus allongé que les Cornish. Ies 4 groupes sexe-souche étaient élevés ensemble et ont reçu un seul aliment, de type engraissement, de l'éclosion à l'abattage. Nos animaux provenaient de 3 lots, nés en octobre 1962 . Ils ont été choisis au hasard parmi les poids moyens de leur groupe respectif et abattus au mêne âge, I I semaines, après un jeûne complet d'environ i 5 heures.

Le poids vif a été noté immédiatement avant l'abattage, puis les animaux étaient saignés, échaudés, plumés et effilés. Le poulet effilé correspond à la carcasse plumée moins l'ensemble intestins + pancréas. Lc poids effilé a été noté après environ 8 heures de ressuyage ì la température du laboratoire. Ies carcasses étaient alors enveloppées dans une feuille d'aluminium et placées au réfrigératcur $\grave{\mathrm{a}}+4^{\circ} \mathrm{C}$ jusqu'il leur utilisation. I'emballage dans l'aluminium permet de supprimer presque complètement les peries par évaporation au cours de la conservation. Dans le cas présent, le temps moyen passé au réfrigérateur a été de 4 jours et la perte moyenne par poulet de $2.8 \mathrm{~g}$, ce qui représentait 0,2 p. ıoo du poirls de la carcasse effilée.

Le jour de la dissection, la tête était coupée au ras du crâne et les pattes à l'articulation tibiotarse-tarsométatarse. Ia graisse "abdominale » et tous les viscères étaient enlevés, obtenant ainsi la carcasse éviscérée. La graisse abdominale, pesée à part, est une graisse de dépôt située contre la paroi interne de l'abdomen et autour du gésier. Nous avons également pesé séparément les " abats consommables ", à savoir le cœur, le gésier nettoyé et le foie sans vésicule biliaire. 
La carcasse éviscérée était ensuite découpée en 4 parties principales : vienne

-- ensemble des membres postérieurs (cuisses et pilons) disséqués au ras de la ceinture pel-

- ensemble des ailes, séparées du tronc au niveau de la ceinture scapulaire ;

- ensemble des blancs, comprenant les muscles pectoraux extérieurs à la cage thoracique : pectoralis superficialis, pectoralis profondus, supracoracoid et partie externe des coracobrachialis ventralis, selon la nomenclature de CinAMBERLAIN (I943);

- reste de la carcasse, représentant la carcasse éviscérée moins les membres et les blancs.

Chaque ensemble autre que les blancs a été disséqué en ses éléments principaux : peau, os, viande, et gros tendons pour les membres; peau, os, viande et divers pour le reste de carcasse. Ici, la viande est composée des muscles et de la graisse inter- et intramusculaire. Dans le reste de carcasce, le groupe "divers " comprend des glandes (thymus, thyroide, glande uropygienne) et des éléments tels que gros nerfs, vaisseaux, caillots de sang, etc. La différence entre le poids de chaque partie de la carcasse avant dissection et la somme des éléments réellement pesés constituait les "pertés de dissection ". Ces dernières paraissent provenir essentiellement de l'élément viande : pertes d'eau par évaporation, graisse intermusculaire, suc cellulaire et débris de muscles restés sur les doigts et la planche ż découper. Or, elles augmentent avec la difficulté des dissections. Én moyenne, nous avions une perte de 6 , o g. pour les cuisses et pilons, ce qui représentait $ז, 6 \mathrm{p}$. Ioo du poids total cuisses + pilons ; pour les ailes, les chiffres correspondants étaient de $4,2 \mathrm{~g}$ et $3, \mathrm{I} \mathrm{p}$. 100 et pour le reste de carcasse de $10,4 \mathrm{~g}$ et $3,4 \mathrm{p}$. Ioo. Afin d'obtenir des rendements en viande plus représentatifs, nous avons inclus les pertes de dissection dans l'élément viande.

Les pesées étaient faites à $5 \mathrm{~g}$ près pour le poids vif, au gramme pour la carcasse globale et au décigramme pour chaque élément de carcasse. Le temps de travail au laboratoire pour la dissection complète de la carcasse effilée était de l'ordre de 5 heures par poulet.

\section{RÉSULTATS}

Dans le tableau I sont résumés les résultats d'abattage : poids vif avant abattage, poids de la carcasse effilée et éviscérée, rendements effilé/vif, eviscéré/vif et eviscéré/effilé.

TABI,EAU I

Résultats d'abatlage

\begin{tabular}{|c|c|c|c|c|c|c|}
\hline \multirow{2}{*}{ Éléments } & \multicolumn{4}{|c|}{ Moyennes par groupe } & \multicolumn{2}{|c|}{ Tous animaux } \\
\hline & $\sigma \mathrm{A}$ & o $\mathrm{A}$ & $\therefore \mathrm{B}$ & q B & Moyeme & Extrênes \\
\hline Poids moyen en $g$ & & & & & & \\
\hline Poulet vivant ......... & 2088 & 1707 & 1635 & 1215 & 1601 & $1160-2145$ \\
\hline Carcasse effilèe $\ldots$ & 1761 & 1391 & 1327 & 978 & $136^{\prime} \mathrm{x}$ & $938-1788$ \\
\hline $\begin{array}{l}\text { Carcasse éviscérée ....... } \\
\quad \text { Rendements en p. } 100\end{array}$ & $1: 30$ & 1122 & 1057 & 772 & 1095 & $730-144^{\prime 2}$ \\
\hline Effilé/vif ............ & 81,\}^{\prime}$ & 81,5 & 81,2 & 80,5 & 81,9 & $79,4-85,5$ \\
\hline Éviscéré/vif . . . . . . . . . . & 68,5 & 65,7 & 64,7 & 63,5 & 6,6 & (6), $3-69,9$ \\
\hline Éviscéré/effilé. ............ & 81,2 & 80,6 & 79,7 & 78,9 & 80,2 & $77,9-81,8$ \\
\hline
\end{tabular}

$I_{1}$ a différence de poids entre les 2 souches étudiées est considérable puisqu'elle approche $500 \mathrm{~g}$, environ 2 écarts-types. Ia raison en est que la souche A, de type "mâle-chair », est fortement sélectionnée pour la croissance alors que les caractères 
économiques n'entrent pas en ligne de compte pour le maintien de la souche $B$, purement expérimentale. En moyenne, le poids effilé représente un peu plus de80 p. roo et le poids éviscéré un peu moins des deux-tiers du poids vif. Le tableau I montre en outre une variation dans le mênue sens des 3 rendements calculés : la. souche $A$ est meilleure que la souche $B$ et les mâles mejlleurs que les femelles.

Le tableau 2 indique la part relative des grandes parties de la carcasse par rapport au poids vif, au poids effilé et au poids éviscéré.

TABI,EAU 2

Répariition des principales parties de la carcasse

\begin{tabular}{|c|c|c|c|c|c|c|}
\hline \multirow{2}{*}{ Éléments } & \multicolumn{4}{|c|}{ Moyennes par groupe } & \multicolumn{2}{|c|}{ Tous animaux } \\
\hline & $\partial \mathrm{A}$ & 우 A & $\partial \mathrm{B}$ & 우 13 & Moyenne & Extrêmes \\
\hline
\end{tabular}

$1^{\circ}$ En p. 100 du poids vif

\begin{tabular}{|c|c|c|c|c|c|c|}
\hline Cuisses + pilons & 25,5 & 23,8 & 24,7 & 22,6 & 24,2 & $22,3-26,7$ \\
\hline Ailes . . . . . . . . . & 8,8 & 8,4 & 8,0 & 8,2 & 8,3 & $7,7-9,1$ \\
\hline Blancs ....... & 11,3 & $11^{\prime}, 1$ & 12,6 & 12,9 & 13,5 & $12,3-14,8$ \\
\hline Reste currcasse $\ldots \ldots \ldots \ldots$ & 19,7 & 19,2 & 18,8 & 19,5 & 19,3 & $18,4-20,0$ \\
\hline Abats consommiables ..... & 3,6 & 3,8 & 4,0 & 4,2 & 3,9 & $3,2-4,4$ \\
\hline Graisse abdominale . & 0,6 & 1,2 & 0,7 & 1,6 & 1,0 & $0,4-2,0$ \\
\hline Pertes abattage $\left({ }^{1}\right) \ldots \ldots \ldots$ & 15,6 & 18,5 & 18,8 & 19,5 & 18,1 & $14,5-20,6$ \\
\hline Divers $\left({ }^{2}\right) \ldots \ldots \ldots \ldots$ & 11,9 & 11,0 & $12, \pm$ & 11,5 & 11,7 & $10,5-12,8$ \\
\hline
\end{tabular}

2o En p. $100 \mathrm{du}$ poids effilé

\begin{tabular}{|c|c|c|c|c|c|c|}
\hline Cuisses + pilons & 30,2 & 29,2 & 30,5 & 28,1 & 29,5 & $27,9-31,3$ \\
\hline Ailes...$\ldots \ldots \ldots$. & $10,{ }^{\prime} \mathrm{k}$ & 10,3 & 9,9 & 10,2 & 10,2 & $9,5-10,9$ \\
\hline Blancs $\ldots \ldots$. & 17,0 & 17,3 & 15,5 & 16,1 & 16,5 & $15,2-17,8$ \\
\hline Reste carcasse $\ldots \ldots \ldots \ldots$ & 23,3 & 23,5 & 23,2 & $2 t^{\prime}, 2$ & 23,6 & $22,7-2,4,8$ \\
\hline Abats consommables .... & 4,3 & ${ }_{4}^{4}, 6$ & 4,9 & 5,2 & 4,8 & $3,7-5,4$ \\
\hline Graisse abdominale .. & 0,7 & 1,5 & 0,9 & 2,0 & 1,3 & $0,5-2,5$ \\
\hline Divers $\left({ }^{2}\right) \ldots \ldots \ldots$ & $1 i, 1$ & 13,6 & 15,1 & 14,2 & $1 /, 1$ & $12,8-15,7$ \\
\hline
\end{tabular}

$3^{\circ}$ En p. 100 dil poids éviscéré

\begin{tabular}{|c|c|c|c|c|c|c|}
\hline Cuisses + pilons . . . . . . & 37,2 & 36,2 & 38,2 & 35,6 & 36,8 & $35,2-38,7$ \\
\hline Ailes $\ldots \ldots \ldots \ldots$ & 12,8 & 12,9 & 12,4 & 12,9 & 12,7 & $12,1-13,7$ \\
\hline Blancs..... . & 21,0 & 21,4 & 19,5 & 20,3 & 20,6 & $19,3-2 \cdot 2,1$ \\
\hline Reste carcasse & $\because 8,7$ & 29,2 & 29,1 & 30,7 & 29,4 & $28,1-31,7$ \\
\hline Pertes découpe.. & 0,3 & 0,3 & 0,8 & 0,5 & 0,5 & $0,2-1,3$ \\
\hline
\end{tabular}

${ }^{(1)}=$ Sang + pluncs + effilage + pertes ressurage.

$\left({ }^{2}\right)=$ Abats et viscères non consommables + pertes découpe.

En moyenne, l'ensemble cuisses et pilons représente près du quart du poids vif, 30 p. Ioo du poids efflé et 37 p. Ioo du poids éviscéré. Pour les blanes, autre partie très appréciée dans un pouiet, les pourcentages respectifs sont voisins de I4, I7 et 2 I p. Ioo; pcur le total ailes + reste de carcasse, ils sont de 28,34 et 42 p. roo. 
Notons enfin que les pertes à l'abattage et les viscères non consommables représentent près de $30 \mathrm{p}$. roo du poids vif, les abats consommables $4 \mathrm{p}$. Ioo et la graisse abdominale seulement I p. Ioo.

Dans le détail des groupes, le tableau 2 montre que les blancs sont toujours plus importants pour la souche A que pour la souche B et les cuisses et pilons plus importants chez les coquelets que chez les poulettes. I.a souche $\mathrm{B}$ et les poulettes ont plus d'abats consommables et de graisse abdominale. Pour les autres éléments, les différences entre groupes évoluent selon le poids de référence considéré : le poids relatif des membres est plus fort pour la souche A que pour la souche B quand on se rapporte au poids vif, mais les différences sont faibles par rapport aux poids effilé et éviscéré ; l'importance des blancs et du reste de carcasse est plus grande chez les femelles que chez les mâles quand on se rapporte au poids effilé ou éviscéré alors que les différences étaient faibles par rapport au poids vif.

Dans le tableau 3, nous avons indiqué la répartition de l'os, de la viande et de la peau, disséqués dans les différentes parties de la carcasse éviscérée, également par rapport au poids vif, au poids effilé et au poids éviscéré.

Dans l'ensemble, la viande de la carcasse représente $45 \mathrm{p}$. Ioo du poids vif, 55 p. Ioo de la carcasse effilée et 69 p. Ioo de la carcasse éviscérée. Pour l'os, les pourcentages sont respectivement de Ir, I4, et I7 p. I0o ; pour la peau, ils sont de 6 et demi, 8 et Io p. Ioo.

La comparaison des groupes montre que les coquelets ont un pourcentage d'os plus fort et un pourcentage de peau plus faible que les poulettes, tandis que pour un même sexe, les différences entre souches sont faibles. Pour la viande totale, les différences sont nettes quand on se rapporte au poids vif (souche $A$ meilleure que la souche $B$ et coquelets meilleurs que les poulettes), mais deviennent peu importantes si on se réfère at poids éviscéré. Le détail de la répartition montre que dans tous les cas la souche $\mathrm{A}$ présente un plus fort pourcentage de blanes que la souche $\mathrm{B}$ et que les coquelets ont plus de viande au niveau des cuisses et dc.j pilons que les poulettes. En passant de la référence poifs vif à la référence poids éviscéré, la supériorité de la souche $A$ pour la viande au niveau des membres s'amenuise, la souche $B$ a plus de viande sur le reste de la carcasse que la souche $A$, les poulettes ont plus de blancs et de viande sur le reste de carcasse que les coquelets.

La répartition des tissus selon les différentes parties de la carcasse est mieux mise en lumière dans le tableau 4 où le total de chaque tissu est supposé égal à roo.

Dans l'ensemble, on constate que le reste de carcasse renferme presque la moitié (47 p. Ioo) de l'os et de la peau de la carcasse éviscérée complète. On trouve presque autant de peau sur les ailes ( 25 p. IOO) que sur les cuisses et les pilons ( 28 p. IOO). I es cuisses et pilons contiennent la plus grande partie de la viande totale (39 p. roo), suivis des blancs (30 p. IOO), du reste de carcasse (22 p. IOO) et des ailes (IO p. IOO).

La comparaison des groupes montre que la répartition de l'os et celle de la peau varient relativement peu en comparaison de la viande : la souche $A$ a plus de blancs que la souche $B$, mais moins de viande sur le reste de la carcasse ; les coquelets ont moins de blancs que les poulettes mais plus de viande au niveau des cuisses et pilons et du reste de carcasse.

Dans le tableau 5, nous indiquons la répartition des tissus dans les cuisses et pilons, les ailes et le reste de carcasse.

C'est l'ensemble cuisses + pilons qui est le plus riche en viande et, corrélati- 
Répartition des tissus de la car.

\begin{tabular}{|c|c|c|c|c|c|c|}
\hline \multirow{3}{*}{ Eléments } & \multicolumn{6}{|c|}{ En p. 100 du poids vif } \\
\hline & \multicolumn{4}{|c|}{ Moyennes par groupe } & \multicolumn{2}{|c|}{ Tous animaux } \\
\hline & $\mathrm{O}^{*} \mathrm{~A}$ & qA & $a \mathrm{~B}$ & \& & Moyenne & Extrimes \\
\hline \multicolumn{7}{|l|}{ Ensemble carcasse éviscérée } \\
\hline Os total $\ldots . .$. & 11,9 & 10,9 & 11,9 & 10,5 & 11,3 & $10,4-12,7$ \\
\hline Viande totale $(\stackrel{1}{)})$ & 47,1 & 45,1 & 43,9 & 43,8 & $4.5,0$ & $42,5-48,6$ \\
\hline Peau totale $\ldots \ldots \ldots \ldots \ldots \ldots \ldots$. & 6,4 & 6,8 & 6,1 & 6,8 & 6,5 & $5,7 \ldots 7,3$ \\
\hline Divers $\left({ }^{2}\right) \ldots \ldots \ldots \ldots \ldots \ldots \ldots \ldots$ & 3,1 & 2,9 & 2,8 & 2,4 & 2,8 & $2,2-3,7$ \\
\hline \multicolumn{7}{|l|}{ Riépartition des os } \\
\hline Cuisses + pilons................. & 4,1 & 3,8 & 4,2 & 3,5 & 3,9 & $3,4-4,6$ \\
\hline Ailes $\ldots \ldots \ldots \ldots \ldots$ & 2,3 & 2,0 & 2,2 & 2,0 & 2,1 & $2,0-2,3$ \\
\hline Reste carcasse $\ldots \ldots \ldots \ldots \ldots \ldots \ldots$ & 5,5 & 5,1 & 5,5 & 5,0 & 4,3 & $4,9-5,8$ \\
\hline \multicolumn{7}{|l|}{ Répartition de la viande } \\
\hline Blancs.$\ldots \ldots \ldots \ldots \ldots \ldots \ldots \ldots \ldots \ldots$ & 14,3 & 14,1 & 12,6 & 12,9 & 13,5 & $12,3-14,8$ \\
\hline Cuisses + pilons $\ldots \ldots \ldots \ldots \ldots \ldots \ldots \ldots \ldots \ldots \ldots \ldots \ldots \ldots$ & 18,5 & 17,2 & 17,9 & 16,3 & 17,5 & $15,9-19,2$ \\
\hline Ailes $\ldots \ldots \ldots \ldots \ldots \ldots \ldots \ldots \ldots \ldots \ldots \ldots \ldots \ldots \ldots \ldots \ldots \ldots \ldots$ & 4,6 & $4, l_{4}$ & ${ }^{4}, 0$ & 4,3 & 4,3 & $3,8-4,8$ \\
\hline Reste carcasse $\ldots \ldots \ldots \ldots \ldots \ldots \ldots \ldots \ldots \ldots \ldots \ldots \ldots \ldots \ldots$ & 9,7 & 9,5 & 9,5 & 10,3 & 9,7 & $8,9-10,9$ \\
\hline Répartition de la peau & & & & & & \\
\hline Cuisses + pilons $\ldots \ldots \ldots \ldots \ldots \ldots \ldots$ & 1,8 & 1,9 & 1,7 & 1,9 & 1,8 & $1,5-2,0$ \\
\hline Ailes $\ldots \ldots \ldots \ldots \ldots \ldots \ldots \ldots \ldots \ldots \ldots \ldots \ldots \ldots \ldots$ & 1,6 & 1,8 & 1,5 & 1,6 & 1,7 & $1,3-2,1$ \\
\hline Reste carcasse $\ldots \ldots \ldots \ldots \ldots \ldots \ldots \ldots \ldots \ldots \ldots \ldots \ldots \ldots \ldots \ldots$ & 3,0 & 3,1 & 2,9 & 3,1 & 3,1 & $2,6-3,4$ \\
\hline
\end{tabular}

(1) Comprend les muscles, la graisse inter- et intranusculaire ainsi que les pertes de dissection.

(2) Représente lcs gros tendons, les thymus et la thyroïde, des déchets divers et les pertes de découpe de la ca

vement, le plus pauvre en peau et tissus osseux. Pour les ailes et le reste de carcasse, la viande représente environ la moitié du poids total; les ailes ont un peu plus de peau et un peu moins d'os que le reste de carcasse.

I a comparaison des groupes montre que le pourcentage d'os est toujours plus élevé chez les mâles que chez les femelles alors que c'est l'inverse en ce qui concerne la peau. Le pourcentage de viande varie très peu au niveau des cuisses et pilons. Pour les ailes, les coquelets B sont inférieurs aux 3 autres groupes. La viande du reste de carcasse est plus importante dans la souche $B$ que dans la souche $A$ et plus importante chez les femelles que chez les mâles. I,e plus grand pourcentage de l'élément " divers " observé pour la souche A tient au fait que les thymus étaient plus développés dans cette souche.

\section{DISCUSSION}

La méthode que nous avons expérimentée permet de bien étudier la répartition des différents éléments de la carcasse, mais elle est longue et ne permet pas d'étudier simultanément un grand nombre d'animaux.

Dans notre essai, le choix des animaux parmi les poids moyens de chaque groupe 
rée par rapport au poulet total

\begin{tabular}{|c|c|c|c|c|c|c|c|c|c|c|}
\hline \multicolumn{5}{|c|}{ En p. $100 \mathrm{du}$ poids effilé } & \multicolumn{6}{|c|}{ En p. 100 du poids éviscéré } \\
\hline \multicolumn{3}{|c|}{ yennes par groupe } & \multicolumn{2}{|c|}{ Tous animaux } & \multicolumn{4}{|c|}{ Moyennes par groupe } & \multicolumn{2}{|c|}{ Tous animaux } \\
\hline 우 A & $\partial \mathrm{B}$ & 우 B & Moyenne & Extrêmes & $\sigma \mathrm{A}$ & 우 $\mathrm{A}$ & of B & 우 B & Moyenne & Extrêmes \\
\hline 13,4 & 14,6 & 13,0 & 13,8 & $12,7-15,5$ & 17,4 & 16,7 & 18,3 & 16,6 & 17,2 & $15,7-19,4$ \\
\hline 55,4 & 54,1 & 54,5 & 54,9 & $52,7-56,9$ & 68,7 & 68,7 & 68,0 & 69,0 & 68,6 & $66,1-70,5$ \\
\hline 8,4 & 7,5 & 8,5 & 8,0 & $7,0-9,2$ & 9,4 & 10,4 & 9,5 & 10,7 & 10,0 & $8,7-11,6$ \\
\hline 3,4 & 3,5 & 2,9 & 3,5 & $2,8-4,4$ & 4,5 & 4,2 & 4,2 & 3,7 & 4,2 & $3,4-5,5$ \\
\hline 4,6 & 5,1 & 4,3 & 4,7 & $4,2-5,6$ & 6,0 & 5,7 & 6,4 & 5,5 & 5,9 & $5,2-7,0$ \\
\hline 2,5 & 2,7 & 2,5 & 2,6 & $2,4-2,9$ & 3,3 & 3,1 & 3,4 & 3,2 & 3,3 & $3,0-$ \\
\hline 6,3 & 6,8 & 6,2 & 6,5 & $6,0-7,1$ & 8,1 & 7,8 & 8,5 & 7,9 & 8,1 & $7,4-8,9$ \\
\hline 17,3 & 15,5 & 16,1 & 16.5 & $15,2-17,8$ & 21,0 & 21,4 & 19,5 & 20,3 & 20,6 & $19,3-22,1$ \\
\hline 21,1 & 21,9 & 20,3 & 21,3 & $20,0-22,5$ & 26,9 & 26,2 & 27,6 & 25,8 & 26,6 & $25,1-27,9$ \\
\hline 5,4 & 4,9 & 5,3 & 5,2 & $4,7-5,7$ & 6,6 & 6,6 & 6,2 & 6,7 & 6,5 & $5,8-7,0$ \\
\hline 11,7 & 11,7 & 12,8 & 11,9 & $10,8-13,5$ & 14,1 & $14,4_{4}$ & 14,6 & 16,2 & 14,8 & $13,4-17,2$ \\
\hline 2,3 & 2,1 & 2,4 & 2,2 & $1,8-2,5$ & 2,6 & 2,9 & 2,6 & 3,0 & 2,8 & $2,3-3,1$ \\
\hline 2,2 & 1,9 & 2,0 & 2,0 & $1,7-2,6$ & 2,4 & 2,7 & 2,4 & 2,6 & 2,5 & $2,1-3,3$ \\
\hline 3,9 & 3,6 & 4,1 & 3,8 & $3,2-4,2$ & 4,4 & 4,8 & 4,5 & 5,1 & 4,7 & $4,0-5,3$ \\
\hline
\end{tabular}

parties principales.

sexe-souche ne rend pas compte de la variabilité réelle qui peut exister à l'intérieur d'un groupe. En conséquence, nous n'avons pas fait d'analyse statistique précise. Nous avons seulement indiqué les valeurs extrêmes observées. Des données plus nombreuses, en cours d'élaboration, nous permettront d'étudier avec plus de précision la variabilité de la composition anatomique du poulet. Nous ferons seulement quelques remarques sur les tendances qui se dégagent de nos résultats et qui confirment, généralement, les observations faites par de nombreux auteurs.

Les moyennes calculées sur l'ensemble de nos animaux permettent d'avoir une idée des rendements obtenus avec le poulet.

La carcasse effilée représente un peu plus des quatre cinquièmes du poids vif et la carcasse éviscérée les quatre-cinquièmes de la carcasse effilée (tabl. I). La viande, élément qui intéresse le plus le consommateur, représente presque la moitié - $45 \mathrm{p}$. Ioo - du poids vif et plus des deux tiers - $69 \mathrm{p}$. Ioo - du poids éviscéré (tab1. 3). Une part importante de cette viande $(68,8$ p. Ioo) est localisée au niveau des blancs et des membres postérieurs (tabl. 4). On peut la comparer aux morceaux dits " de première catégorie " chez les animaux de boucherie. Une dissection simple des muscles pectoraux, des cuisses et des pilons permet d'en apprécier l'importance (cas du travail d'OKERMAN, I960).

Considérons plus spécialement la carcasse effilée, qui est la forme la plus courante 
TABLEAU 4

Répartition des éléments de chaque tissu par rapport au tissu tolal

\begin{tabular}{|c|c|c|c|c|c|c|}
\hline \multirow{2}{*}{ Éléments } & \multicolumn{4}{|c|}{ Moyennes par groupe } & \multicolumn{2}{|c|}{ 'Tous animaux } \\
\hline & o $A$ & 우 A & o $\mathrm{B}$ & 우 $\mathrm{B}$ & Moyenne & Extrêmes \\
\hline Os & & & & & & \\
\hline Cuisses + pilons...$\ldots \ldots$ & 34,3 & $3 t, 3$ & 35,1 & 33,1 & 34,2 & $31,6-37,0$ \\
\hline 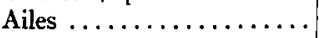 & 19,2 & 18,6 & 18,7 & 19,3 & 18,9 & $18,0-19,8$ \\
\hline $\begin{array}{c}\text { Reste carcasse } \ldots \ldots \ldots \\
\text { Viande }\end{array}$ & 46,6 & 47,0 & 46,3 & 47,6 & 46,9 & $45,0-48,7$ \\
\hline Blancs $\ldots \ldots \ldots \ldots \ldots$ & 30,5 & 31,2 & 28,7 & 29,5 & 30,0 & $28,2-31,9$ \\
\hline Cuisses + pilons $\ldots \ldots \ldots$ & 39,2 & 38,1 & 40,6 & 37,3 & 38,8 & $36,3-41,3$ \\
\hline Ailes $\ldots \ldots \ldots \ldots \ldots \ldots$ & 9,7 & 9,7 & 9,1 & 9,7 & 9,6 & $8,8-10,2$ \\
\hline $\begin{array}{c}\text { Reste carcasse } \ldots \ldots \ldots \\
\text { Peau }\end{array}$ & 20,6 & 21,0 & 21,5 & 23,5 & 21,7 & $20,2-21,9$ \\
\hline Cuisses + pilons ......... & 27,6 & 27,8 & 27,2 & 28,2 & 27,7 & $26,0-29,1$ \\
\hline Ailes...$\ldots \ldots \ldots \ldots \ldots$ & 25,6 & 26,3 & 25,1 & 24,0 & 25,3 & $21,4-28,6$ \\
\hline Reste carcasse ......... & 46,8 & 16,0 & 47,6 & 47,8 & 47,1 & $45,1-50,2$ \\
\hline
\end{tabular}

TABLEAU 5

Répartition des tissus à l'intérieur de chaque partie de la carcasse (en p. Ioo du poids de chaque partie)

\begin{tabular}{|c|c|c|c|c|c|c|}
\hline \multirow{2}{*}{ Éléments } & \multicolumn{4}{|c|}{ Moyennes par groupe } & \multicolumn{2}{|c|}{ Tous animaux } \\
\hline & ô $A$ & 우 $\mathrm{A}$ & o $\mathrm{B}$ & 우 B & Moyenne & Extrêmes \\
\hline Cuisses + pilons & & & & & & \\
\hline Os $\ldots \ldots \ldots \ldots \ldots \ldots \ldots$ & 16,1 & 15,8 & 16,8 & 15,4 & 16,0 & $14,0-18,1$ \\
\hline Viande $\ldots \ldots \ldots \ldots \ldots$ & 72,5 & 72,2 & 72,2 & 72,3 & 72,3 & $70,4-74,8$ \\
\hline Peau $\ldots \ldots \ldots \ldots \ldots \ldots$ & 7,0 & 7,9 & 6,8 & 8,5 & 7,5 & $6,0-8,8$ \\
\hline $\begin{array}{c}\text { Divers }\left({ }^{1}\right) \ldots \ldots \ldots \ldots \\
\text { Ailes }\end{array}$ & 4,4 & 4,1 & 4,2 & 3,8 & 4,2 & $3,4-4,8$ \\
\hline Os $\ldots \ldots \ldots \ldots \ldots \ldots \ldots \ldots$ & 26,1 & 24,2 & 27,6 & $2 \prime^{\prime}, 8$ & 25,6 & $23,5-28,7$ \\
\hline Viande $\ldots \ldots \ldots \ldots \ldots$ & 51,9 & 51,9 & 50,0 & 52,0 & 51,5 & $46,8-53,5$ \\
\hline Peau...$\ldots \ldots \ldots \ldots$ & 18,8 & 21,3 & 19,2 & 20,0 & 19,8 & $17,5-24,2$ \\
\hline $\begin{array}{c}\text { Divers }(\mathbf{1}) \ldots \ldots \ldots \ldots \\
\text { Reste carcasse }\end{array}$ & 3,2 & 2,6 & 3,2 & 3,2 & 3,1 & $2,4-3,8$ \\
\hline Os $\ldots \ldots \ldots \ldots \ldots \ldots \ldots$ & 28,2 & 26,9 & 29,2 & 25,7 & 27,5 & $24,8-31,5$ \\
\hline Viande $\left({ }^{2}\right) \ldots \ldots \ldots \ldots$ & 49,3 & 49,6 & 50,4 & 52,7 & 50,5 & $45,7-54,3$ \\
\hline Peau....$\ldots \ldots \ldots \ldots$ & 15,3 & 16,4 & 15,5 & 16,8 & 16,0 & $14,1-17,4$ \\
\hline Divers $\left({ }^{a}\right) \ldots \ldots \ldots \ldots$ & 7,2 & 7,1 & 4,9 & 4,8 & 6,0 & $2,7-10,1$ \\
\hline
\end{tabular}

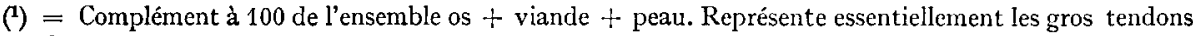
des membres.

$\left({ }^{2}\right)=$ Blancs non compris.

$\left({ }^{3}\right)=$ Représente essentiellement les glandes du cou (thymus, thyroide) et divers déchets (gros nerfs, vaisseaux, etc.). 
sous laquelle se vend le poulet en France. Les tableaux 2 et 3 montrent que le total des éléments consommables constituent plus des deux tiers du poids effilé, à savoir :

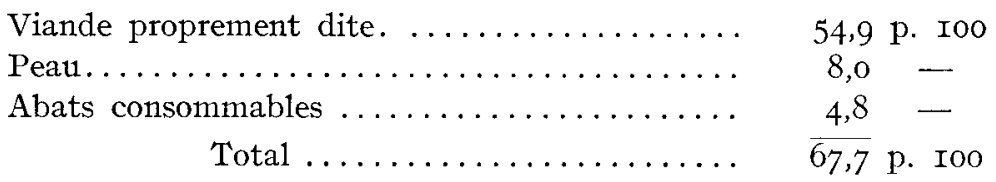

Les viscères non consommables, les os et les déchets obtenus à la dissection représentent environ le tiers du poids effilé.

En ce qui concerne la variabilité entre groupes, nous avons plusieurs fois noté une évolution des différences selon le poids de référence considéré (tab1. 2 et 3). Ce fait est lié aux variations propres du rendement à l'abattage, variations qui sont relativement importantes (tabl. I).

Par rapport à la carcasse éviscérée, les grandes parties de la carcasse et les tissus pris dans leur ensemble présentent une remarquable homcgénéité quand on compare les deux souches, qui sont cependant d'origine et de conformation très différentes. C'est ce que montrent les chiffres suivants tirés des tableaux 2 et 3 :

Moyennes pour les 2 sexes

Cuisses et pilons $\ldots \ldots \ldots \ldots \ldots \ldots$.

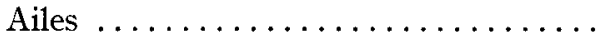

Tronc (y compris les blancs et le cou)

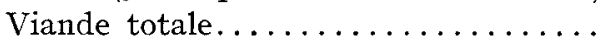

Os total

Peau totale
Souche $A$

36,7 p. Iо0
I2,8 -
$50,1-$
$68,7-$
I7,0 -
$9,9-$

Souche B

36,9 p. I0о
I $2,6=$
$49,8-$
$68,6-$
I7,4 -
I0, I -

Des observations analogues, concernant le rendement en viande consommable totale du poulet, peuvent se faire à partir des données de MAW et MAW (I939), Scotrs et Darrow (I953), MORrison et al. (r954), ORR (I955), Dawson et al. (I958), GILPIN et al. (I960), KONDRA et al. (I962). Ces auteurs comparaient des souches ou des croisements commerciaux élevés dans des conditions semblables. FRY et al. (Ig62) observent également de faibles variations dans le pourcentage des régions corporelles de dindes de souches différentes, de même que Boccard et DUMONT (I960) pour les régions corporelles d'agneaux de poids voisins mais de races très différentes. L'hypothèse d'une " loi d'harmonie anatomique ", proposée par ces derniers auteurs, semble donc s'appliquer également aux souches de volailles.

Nous retrouvons cependant une certaine variabilité si nous nous intéressons à la répartition de la viande selon les différentes parties de la carcasse éviscérée, ainsi que le montrent les chiffres suivants tirés du tableau 3 :

Moyenne pour les 2 sexes

Viande des cuisses et pilons...

Viande des ailes..............

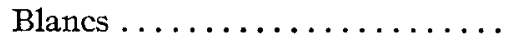

Viande du reste de carcasse...

Total ..........

\section{Souche $A$}

26,6 p. Ioo

$6,6-$

$2 \mathrm{x}, 2-$

$14,3-$

$\overline{68,7}$ p. I00
Souche B

26,7 p. roo

6,5

I9,9

I5,5 -

$\overline{68}, \overline{6}$ p. Iоo 
Dumont et al. (196r) ont noté sur des carcasses de bovins Charolais une variabilité nettement plus grande pour les muscles individuels, ou les groupes musculaires, que pour le poids total des muscles de la carcasse. Ici, la souche $\mathrm{A}$ a plus de blancs mais moins de viande sur le reste de carcasse que la souche B. Au total, les 2 souches s'équilibrent. Les pourcentages de viande au niveau des membres varient peu. Il sera intéressant de préciser ces résultats sur un plus grand nombre de données : on pourrait en déduire que le type "chair " (ici la souche Cornish) est mieux caractérisé par la répartition de la viande que par le pourcentage total de cette viande.

Entre sexes, les différences de rendement sont plus nettes que les différences entre souches. Par rapport à la carcasse éviscérée, nous obtenons les chiffres suivants (tabl. 2 et 3) :

Moyennes pour les 2 souches

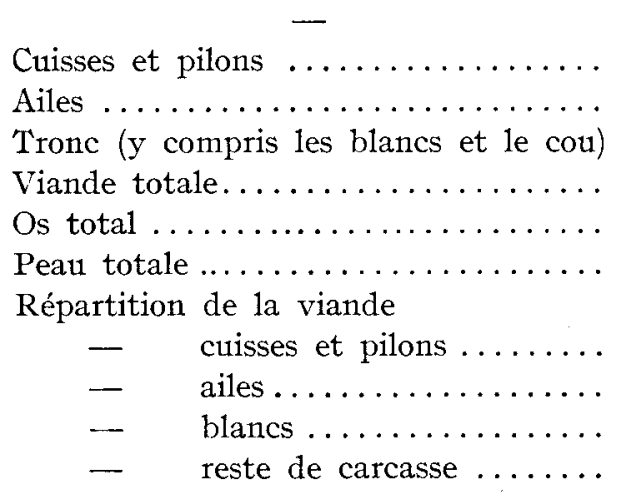

\section{Coquelets}

$\begin{array}{rrrr}37,7 & \text { p. I0o } & 35,9 & \text { p. I00 } \\ \text { I2,6 } & - & \text { I } 2,9 & - \\ 49, \mathrm{I} & - & 50,8 & - \\ 68,3 & - & 68,9 & - \\ \mathrm{I} 7,8 & - & \mathrm{I} 6,6 & - \\ 9,4 & - & \mathrm{I} 0,6 & - \\ & & & \\ 27,3 & - & 26,0 & - \\ 6,4 & - & 6,7 & - \\ 20,2 & - & 20,9 & - \\ \mathrm{I} 4,4 & - & 15,3 & -\end{array}$

La plupart des auteurs font des observations analogues. Il se semble pas que la loi d'harmonie anatomique puisse s'appliquer pour les variations entre mâles et femelles. D'une façon générale, les poulettes ont des cuisses et pilons moins développés et un tronc plus important que les coquelets. Elles ont moins d'os, plus de viande (en particulier plus de blancs), plus de peau et plus de graisse abdominale. Ces deux derniers éléments peuvent être liés dans la mesure où la peau contient également la graisse sous-cutanée, elle-même en corrélation avec la graisse abdominale (GưrTERIDGE, I937). Le rendement à l'abattage des poulettes est plus faible que celui des coquelets (tabl. I), donnant des animaux moins intéressants pour le conditionneur. Pour le consommateur, au contraire, elles présentent une carcasse plus avantageuse.

\title{
Reçu pour publication en juin 1964 .
}

\section{SUMMARY}

\author{
A TRIAL OF DIRECT ESTIMATION OF THE DIFFERENT COMPONENTS \\ OF CHICKEN CARCASSES WITH THE OBJECT OF ASSESSING MEAT YIELD
}

I 2 raw carcasses of I I week-old chickens were completely dissected : 3 males and 3 females each of a Cornish strain and of a strain obtained from White-Bresse and Neze Hampshire breeds. The birds were all reared and fed in the same way. 
In our trial, the mean composition of the chicken was :

P.roo liveweight

Dressing and eviscerating losses $\ldots \ldots \ldots \ldots \ldots \ldots \ldots \ldots \ldots \ldots$

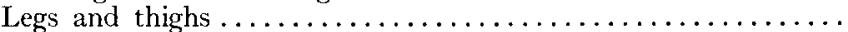

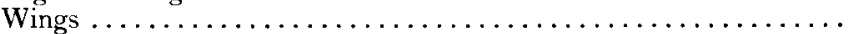

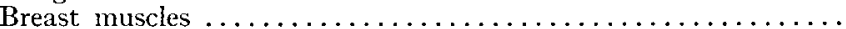

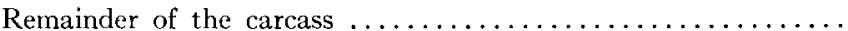

Edible offals (liver + heart + gizzard) $\ldots \ldots \ldots \ldots \ldots \ldots \ldots$.

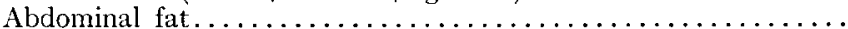

The eviscerated carcass represented two-thirds of the liveweight; on average it had i 7 p. Ioo bone, Io p. Ioo skin and $69 \mathrm{p}$. I oo meat. The complete edible portion, comprising meat, skin and offals was about 55 p. Ioo of liveweight and 68 p. Ioo of the "french dressed weight " (weight after plucking and drawing of the intestines and pancreas).

Slaughter yield was greater in the Cornish strain, and in males. Expressed as percentage of eviscerated carcass there was little variation in the components of the carcass especially between strains. 'The percentage of total meat was the same in both cases, but the meat was better distributed on the carcasses of the Cornish strain, with a greater proportion of breast muscle. Carcasses of pullets had less bone and more breast muscle than did those of cockerels.

\section{RÉFÉREINCES BIBLIOGRAPHIQUES}

Boccard R., Dumont B. L., Ig6o. Étude de la production de la viande chez les ovins. II. Variations de l'importance relative des différentes régions corporelles de l'agneau de boucherie. Ann. Zootech., 9 , $355-363$.

Brown P. B., BeAN H. W., 1952. The yield of edible meat from different market classes of chickens. Poull. Sci., 31, 232-234.

Chamberlain F. W., 1943. Atlas of avian anatomy. Michigan Agric. Exp, Sla. Mem. Bull. 5 .

Dawson L. I.., Walters S., Davidson J. A., 1958. Cooked meat yield from 4 strains of chickens, 6 and 16 weeks of age. Poult. Sci., 39, 227-23o.

Delpecil P., Gouteron J., I962. Croissance du poulet et évolution de la composition chimique des tissus en fonction de l'âge et du poids. Proc. 12th World's Poult, Cong., Syänev, 195-198.

Dumonr B. L., Le Guelte J., Arnoux J., ig6r. Étude biométrique des bovins de boucherie. I. Variabilité de la composition anatomique de la carcasse des bovins charolais. Ann. Zootech., 10, 149-154

Frischknecht C. O., Jull M. A., 1946. Amount of breast meat and live and dressed grades in relation to body measurements in 12 week old purebred and crossbred chickens. Poult. Sci., 25, 330-345.

Fry J. L., Rao O. S., Rasplicka L. D., 1962. Factors affecting the yield of turkeys parts. Poult. Sci., 41, $1299^{-1} 303$.

Gilpin G. L., II Arkin A. M., Redstrom R. A., Dawson E. H., ig6o. Quality and yield of modern and old type chickens. Poull. Sci., 39, 924-93o.

GutTeridge H. S., 1937. Methods and rations for fattening poultry. Sci. Agric., 17, 310-358.

Harsitaw H. M., I $93^{8}$. The effect of fattening at different ages on the composition of cockerels. Poult. Sci., 17, 163-169.

Hathaway H. E., Champagne G. B., Watts A. B., Upp C. W., I953. Meat yield of broilers of different breeds strains and crosses. Poull. Sci., 32, $968-976$.

Haverman H., Wegner R. M., Hartfield W., 1957. Hähnchenmast und Ausschlachtungsversuche mit verschiedenen Rassen und Kreuzungen. Arch. Geflïgelk., 21, I 70-199.

Henderson E. W., I946. Percentage of edible meat in relation to meat type score of chickens, Michigan Agric. Exp. Sta. Quart. Bul., 28, 1 76 -1 80.

Kondra P. A., Riciands J. F., Ilodgson G. C., ig6z. The effect of sex, ration and strain on meat yield and its determination in chicken broilers. Poull. Sci., 41, 922-927.

MaW A. J. G., MAW W. A. I939. The relation of body measurements to the fattening gain and the percentage of edible flesh in the fowl. Sci. Agric., 19, $589^{-5} 59^{6 .}$

Mitchell H. H., Card L. I., Hamilton T. S., I926. The growth of while Plymouth Rock chickens. Illinois Agric. Exp. Sla. Bull. 278.

Mitchell H. H., Card I.. E., Hamilton T. S., 1931. A technical study of the growth of While Legharn chickens. Illinois Agric. Exp. Sta. Bull. 367 . 
Morrison M. A., Sauter E. A., Mc Larren B. A., Stadelman W. J., J., I954. Some factors affecting yield and acceptability of chicken broilers. Poult. Sci., 33, I I 22-I I 25 .

ORR H. L., I955. Effect of strain, sex and diet on dressing percentage and on cooked meat yield of ro week old broilers. Poult. Sci., 34, I093-I097.

Okerman F., I960. Quelques observations faites avec des poulets de différentes races et croisements. Meded. Landbhogesch. Gent., 25, 803-823.

PALSSON H., 1955. Conformation and body composition. In : Hammond, Progress in the phyisology of farm animals, Vol 2, 430-542. Butterworths Scientific Publications, London.

PETERS G. H., I958. Ausschlachtungsversuche bei Geflügel unterschiedlicher Gewischtsklassen unter besonderer Berücksichtigung verschiedener Hühnerassen und Gefügelarten. Arch. Geflügelk., 22, I70-196 et 240-260.

Poley W. E., Moxon A. L., Wilson W. O., Dolecek R. L., I950. Effects of com, wheat and barley in the diet on the physical and chemical composition of fryers and roasters. J. Agric. Res., 61, I6I-I 78 .

RICARD F. H., I96I. Relations entre l'importance du squelette et quelques mesures de conformation chez le poulet de chair. Ann. Zootech., 10, I4I-I42.

Stotis C. E., Darrow M. I., I953. Yields of edible meat from Comish crossbreds, non-cornish crossbreds and purebred broilers. Poult. Sci., 32, I 45-150.

Wilson P. N., I 554. Growth analysis of the domestic fowl. II. Effect of plane of nutrition on carcass composition. J. Agric. Sci., 44, 67-85. 